\title{
Association between toe flexor strength and spatiotemporal gait parameters in community-dwelling older people
}

\author{
Shogo Misu ${ }^{1,2^{*}}$, Takehiko Doi ${ }^{3,4,5}$, Tsuyoshi Asai ${ }^{6}$, Ryuichi Sawa ${ }^{2}$, Kota Tsutsumimoto ${ }^{3,7}$, Sho Nakakubo ${ }^{2,3}$,
} Minoru Yamada ${ }^{8}$ and Rei Ono ${ }^{2}$

\begin{abstract}
Background: The toe flexor muscles perform a crucial function to control foot movement and assist with propulsive force when walking. However, the association between toe flexor strength and spatio-temporal gait parameters is largely unknown. Spatiotemporal gait parameters represent gait characteristics, and are good measures of the functional status and degree of safe ambulation among community-dwelling older adults. Herein, we examined the association between the toe flexor strength and spatiotemporal gait parameters in community-dwelling older adults.

Methods: Ninety-three community-dwelling older people (mean age: $73.2 \pm 4.2$ years, 53 women) participated in this study. The strength of the toe flexor muscles was assessed using a toe strength measuring instrument and a strain gauge. The measurements were performed once on each foot, and the average of the right and left was used in the analysis. Gait analysis was performed on a 15-m walkway under usual- and fast-pace conditions. The medial 10-m walking time was measured and walking speed was calculated. Acceleration and angular velocity of the right heel were measured using a wireless miniature sensor unit and used to compute cadence, percent of swing time in gait cycle (\%swing time), and stride length.
\end{abstract}

Results: In multiple regression analyses adjusted for age, sex, body height, body weight, and hand grip strength, no associations between toe flexor strength and spatiotemporal gait parameters at usual pace were found. Conversely, under the fast-pace condition, decreased toe flexor strength was significantly associated with slower walking speed $(\beta=0.22$, $p=0.049)$, lower\%swing time $(\beta=0.34, p=0.009)$, and shorter stride length $(\beta=0.22, p=0.011)$ after adjustment.

Conclusion: In community-dwelling older people, decreased strength of toe flexor was correlated with slower walking speed, shorter periods of single-limb support phase, and shorter stride length during fast-pace walking. These data provide further support for an important role of toe flexor muscles in walking.

Keywords: Gait, Toe flexor strength, Spatiotemporal gait parameter, Community-dwelling older people

\section{Background}

During walking, the foot is the only source of direct contact with the ground. The foot provides mechanical support for the body and sensory information regarding body position, which contributes to maintaining stability and providing forward propulsion. The toe flexor muscles

\footnotetext{
* Correspondence: s-misu@kobe-nishishimin-hospi.jp

${ }^{1}$ Kobe City Hospital Organization, Kobe City Medical Center, West Hospital, 2-4 Ichibancho, Nagata-ku, Kobe, Hyogo 653-0013, Japan

${ }^{2}$ Department of Community Health Sciences, Kobe University Graduate School of Health Sciences, 7-10-2 Tomogaoka, Suma-ku, Kobe, Hyogo 654-0142, Japan

Full list of author information is available at the end of the article
}

perform a crucial function to control foot movement and assist with the propulsive force when walking. This is achieved by the contraction of the muscles at the late stance phase in the gait cycle [1,2]. By determining conditions for equilibrium from ground force distribution and anthropometrical foot data, it was estimated that the muscles of the flexor hallucis longus, brevis, flexor digitorum longus and brevis exert forces of approximately $52 \%, 36 \%, 9 \%$ and $13 \%$ of body weight during propulsion, respectively [3].

The association between toe flexor strength and objective gait parameters remains unclear, although the role of 
the toe flexor for gait is considered important. Various methods with high reliability have been developed for evaluating the strength of toe flexor muscles, including the paper grip test for measuring the forces under the toes while subjects push down a force-plate using their toes, hand-held dynamometry, and the method using a foot-gripping force meter including a strain-gauge [4-6]. Using these methods, it was demonstrated that aging was associated with reduced toe flexion strength $[4,5]$, and that toe deformities were associated with weak toe flexor strength $[7,8]$. In addition, older people with reduced toe flexor strength had impaired balance function and a higher risk of fall [7,9-11]. Nevertheless, there are few studies that investigated the association between toe flexor strength and gait parameters. Menz et al. [12] reported that toe flexor strength was associated with plantar forces and pressures during walking in older people who resided in a retirement village. Spink et al. [10] reported that hallux plantar flexion strength was associated with walking speed at a normal pace in community-dwelling older people who had an elevated risk of falling and self-reported disabling foot pain. However, these studies did not include spatiotemporal gait parameters except for walking speed. Thus, the effects of toe flexor strength to spatiotemporal gait parameters (e.g., cadence, swing time, and stride length) remain unclear. Spatiotemporal gait parameters are good measures of the functional status and degree of safe ambulation among communitydwelling older adults [1]. Given the function of the toe flexor muscle during gait, we hypothesized that toe flexor strength would be associated with spatiotemporal gait parameters.

The aim of this study was to determine the association between the toe flexor strength and spatiotemporal gait parameters during usual-pace walking and fast-pace walking in community-dwelling older adults. Walking at a fast speed (fast-pace walking) may place a greater demand on physiological systems, and thus provide a good indication of their function [13-15].

\section{Methods}

\section{Participants}

One hundred and twenty community-dwelling older people, aged over 60 years, were recruited through a community organization for older people. Eligibility criteria for this study were that subjects could walk over $15 \mathrm{~m}$ independently without the use of a gait aid and that their feet did not have any orthopedic anomalies that would make it difficult to perform the measurement of toe flexor strength. Participants were excluded if they had neurological disease that would affect gait (e.g., stroke or Parkinson's disease), or cognitive impairment (Japanese version of Rapid Dementia Screening Test score $<8)[16,17]$. Ninety-three people met the criteria and participated in this study. The Research Ethics Committee of the Kobe University Graduate School of Health Science approved the study, and all subjects participating in the study provided informed consent according to the ethical standards set forth in the declaration of Helsinki.

\section{Toe flexor strength and other measures}

The strength of the toe flexor muscles was assessed using a toe strength measuring instrument (T.K.K. 3361; Takei Scientific Instruments, Niigata, Japan) (Figure 1). The apparatus uses a strain gauge to measure the gripping force of the toe flexor muscles. The resolution performance is $0.1 \mathrm{~kg}$. Subjects sat on a chair with the test foot placed on the instrument and the distal phalanx of the great toe and the second to fifth middle phalanxes attached to the gripping bar. Their hip, knee, and ankle were kept at 90 degrees. After sufficient familiarization, subjects were instructed to grip the bar as hard as possible without any other movement of their lower extremity. The measurements were completed once on each foot, and the average of the right and left was used in the analysis. We also measured hand grip strength as the representative measure of general muscle strength to explore the independent effect of toe flexor strength to gait parameters. Hand grip strength was determined using a hand grip dynamometer (T.K.K. 5401; Takei Scientific Instruments). The measurements were completed once on each hand, and the average was used in the analysis.

We assessed the following background characteristics using a questionnaire: age, sex, medical conditions, number of medications, history of falls and participant's life space. The participants' medical conditions, number of medications and fall events during the past 12 months was determined at interviews. Life space was assessed using a life-space assessment (LSA) questionnaire. An LSA assesses frequency of movement (how many days within a week) during the 4 weeks prior to assessment

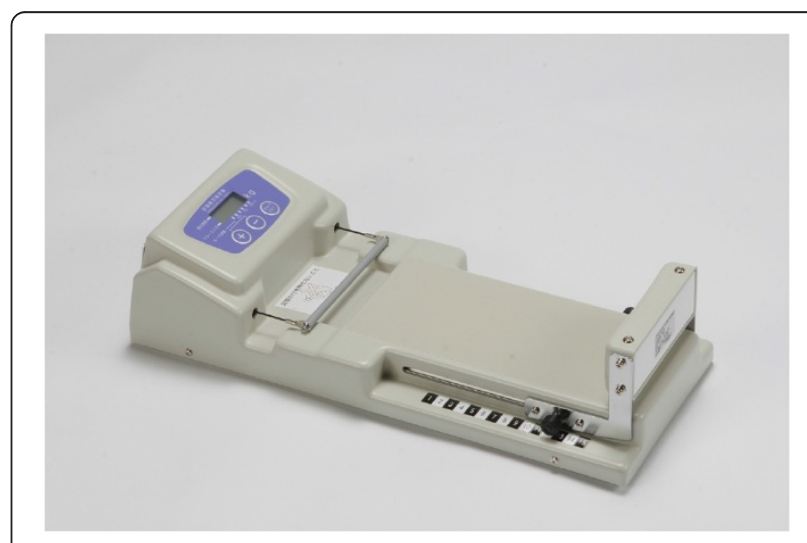

Figure 1 Toe strength measuring instrument. 
for five different life-space levels [18]. From a total of 20 items, a composite score in the range of $0-120$ is computed. Higher scores indicate unrestricted lifespace mobility. The reliability and validity of an LSA in community-dwelling older adults have been previously reported [18]. An LSA reflects physical activity status indirectly in elderly people [19]. The anthropometric index (body weight and body height) was also obtained from physical examination.

\section{Gait procedure and apparatus}

Participants were instructed to walk along a 15-m smooth, horizontal walkway including a $2.5-\mathrm{m}$ space before each end of the walkway for acceleration and deceleration while wearing appropriate-sized shoes checked before each gait assessment. Two walking conditions were measured successively in nonrandomized order to avoid the lying effect whereby the usual-pace would be contaminated by the fast-pace condition: subjects first walked at a selfselected usual pace and then walked as fast as possible without running [13,20-22]. The measurements were performed over the medial 10-m distance. The 10-m walking time was measured, and walking speed was expressed in meters per second.

The wireless miniature sensor unit containing a threeaxis accelerometer and a three-axis gyroscope (MVP-RF8; MicroStone, Nagano, Japan) was attached to the posterior surface of the right heel using surgical tape to compute spatiotemporal gait parameters by identifying heel contact and toe off. The miniature sensor unit minimizes restrictions of walking movements. All signals were sampled at $200 \mathrm{~Hz}$ and synchronously wirelessly transferred to a personal computer via a Bluetooth personal area network.

\section{Data analysis}

Signal processing was performed with commercially available software (MATLAB, Release 2011b; MathWorks, Natick, MA, USA). Before the analysis, all acceleration and angular velocity data were low-pass filtered with a cutoff frequency of $20 \mathrm{~Hz}$. The temporal gait parameters (cadence and percent of swing time in gait cycle; \%swing time) were then determined. On the basis of pilot testing, a heel-contact event was identified as a vertical acceleration peak, while a toe-off event was identified as the maximum heel angular velocity in the sagittal plane. These events were used to calculate temporal gait parameters. Percent of swing time were averaged from five consecutive gait cycles, and were used in the analysis. The average stride length was determined by multiplying gait speed by mean stride time.

\section{Statistical analysis}

Variability of toe flexor strength was transformed into a normal distribution using log transformations because of

a right skewed distribution. To examine the association between toe flexor strength and spatiotemporal gait parameters at both usual and fast pace, Pearson correlation coefficients were first computed. Forced-entry multiple regression analyses were then used to clarify the associations between toe flexor strength and spatiotemporal gait parameters independently of confounders. Within participants characteristics measures, variables associated with the strength of toe flexor $(p<0.10)$ in univariate analysis were forced to include as confounders. The overall statistical significance level was set at 0.05 . All statistical analyses were performed using JMP9.0 J software (SAS Institute Japan, Tokyo, Japan).

\section{Results}

The characteristics of the subjects and spatiotemporal gait parameters are summarized in Table 1 . The mean \pm

Table 1 Subject characteristics and spatiotemporal gait parameters

\begin{tabular}{lcc}
\hline & Mean \pm S.D. & Range \\
\hline Age, years & $73.2 \pm 4.2$ & $63-88$ \\
Sex, women, $\mathrm{n}(\%)$ & $53(57.4)$ & \\
Body weight, $\mathrm{kg}$ & $56.0 \pm 10.8$ & $37.1-88.4$ \\
Body height, cm & $155.3 \pm 8.8$ & $139.7-175.4$ \\
BMl, $\mathrm{kg} / \mathrm{m}^{2}$ & $23.1 \pm 3.2$ & $15.1-35.9$ \\
LSA & $78.8 \pm 18.1$ & $44-120$
\end{tabular}

Falling in past 12 months, $\mathrm{n}(\%) \quad 19$ (20.4)

Medical conditions, $\mathrm{n}(\%)$

Osteoarthritis
Rheumatoid arthritis
Hypertension

$15(16.1)$

Hypertension

$1(1.1)$

Diabetes mellitus

Heart disease

Number of medications, $\mathrm{n}$

Strength of hand grip, $\mathrm{kg}$

Strength of toe flexor, $\mathrm{kg}$

Usual-pace walking

Walking speed, $\mathrm{m} / \mathrm{s}$
Cadence, steps/min
\%Swing time
Stride length, $\mathrm{m}$

Fast-pace waking

$\begin{array}{lcc}\text { Walking speed, } \mathrm{m} / \mathrm{s} & 1.72 \pm 0.23 & 1.04-2.35 \\ \text { Cadence, steps/min } & 137.8 \pm 11.9 & 111.1-168.1 \\ \text { \%Swing time } & 41.61 \pm 2.07 & 35.61-46.50 \\ \text { Stride length, } \mathrm{m} & 1.50 \pm 0.17 & 1.02-1.84\end{array}$

Values are means \pm standard deviation or number of subjects (percentages) as indicated. Spatiotemporal gait parameters were averaged from five consecutive gait cycles. S.D. = Standard deviation; $B M I=$ Body mass index; $L S A=$ Life-space assessment; \%Swing time = Percent of swing time in gait cycle. 
(A) Usual-pace walking
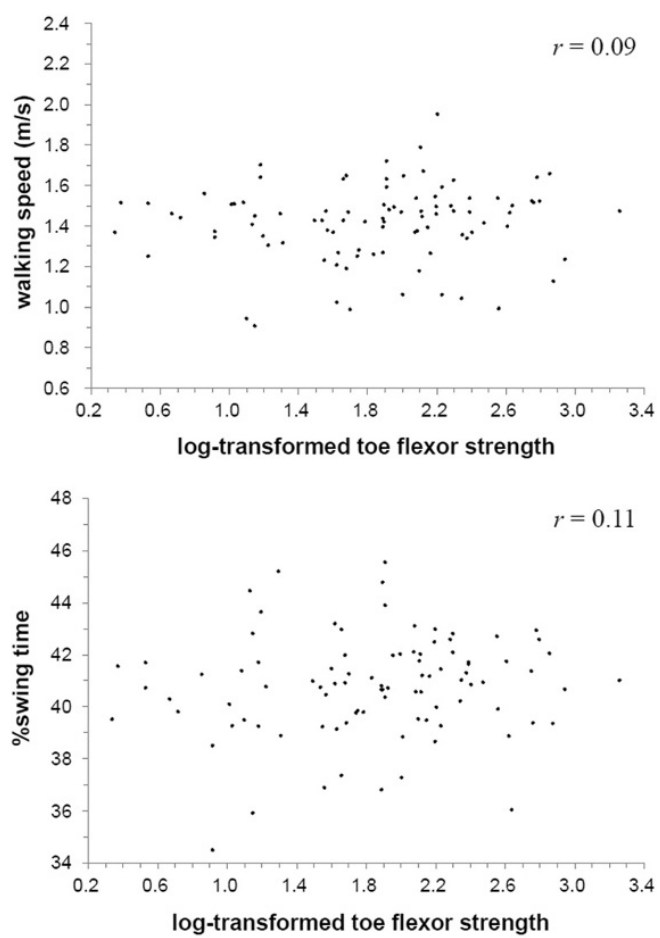

(B) Fast-pace walking
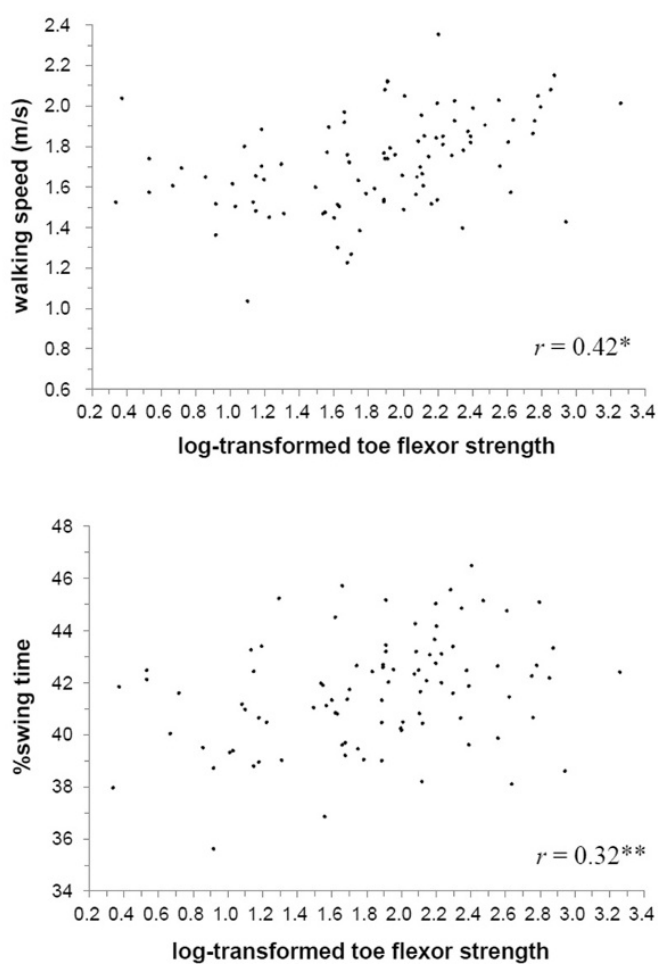
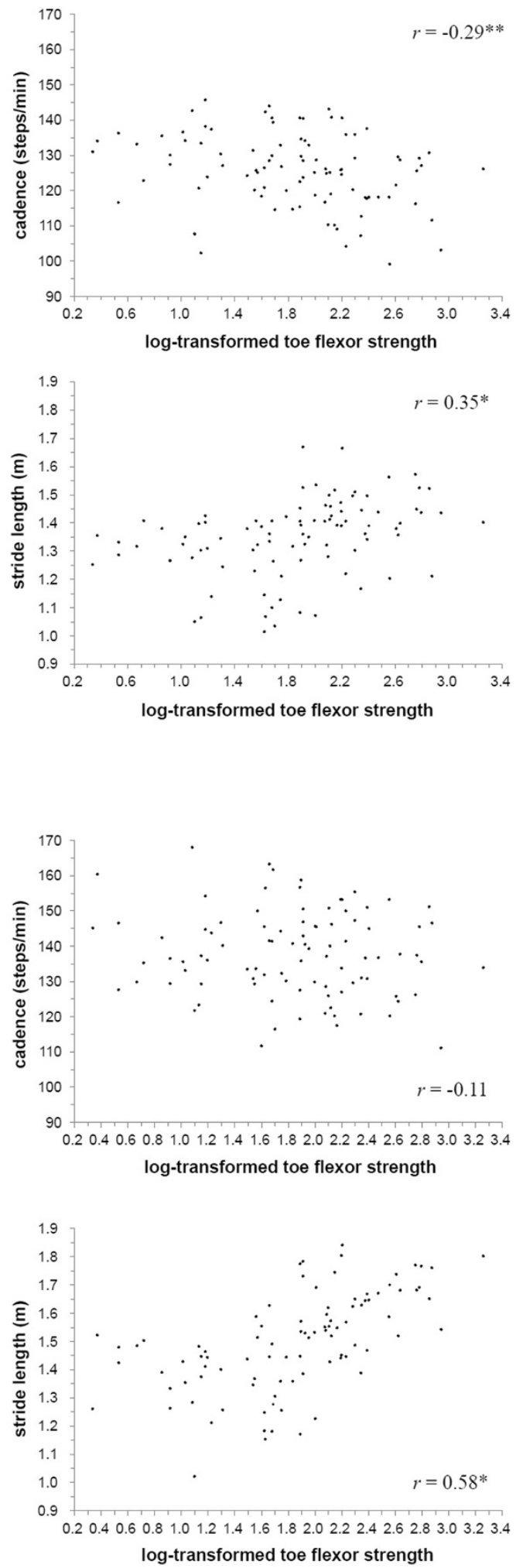

Figure 2 Bivariate correlation between strength of toe flexor and spatiotemporal gait parameters during usual-pace walking (A) and during fast-pace walking (B). Variability of toe flexor strength was performed by log transformations. The described $r$ value represents Pearson correlation coefficients $\left({ }^{*} p<0.001,{ }^{* *} p<0.01,{ }^{* * *} p<0.05\right)$. 
standard deviation (S.D.) of age in our sample was $73.2 \pm 4.2$ years, and $57.4 \%$ of the sample was female. The mean \pm S.D. of walking speed at usual and fast pace were $1.42 \pm 0.19 \mathrm{~m} / \mathrm{s}$ and $1.72 \pm 0.23 \mathrm{~m} / \mathrm{s}$, respectively.

Correlation coefficients representing the association between toe flexor strength and spatiotemporal gait parameters are displayed in Figure 2. For the usual-pace condition, the strength of the toe flexor was negatively associated with cadence $(r=-0.29)$ and positively associated with stride length $(r=0.31)$ (Figure 2A). For the fast-pace condition, the strength of the toe flexor was positively associated with walking speed $(r=0.42)$, \%swing time $(r=0.32)$, and stride length $(r=0.58)$ (Figure 2B).

Table 2 shows the multivariable adjusted associations of spatiotemporal parameters with toe flexor strength. Age, sex, body weight, body height, and hand grip strength were entered into the regression models as confounders as those variables were associated with the strength of toe flexor $(p<0.10)$ in the univariate analysis. After adjustment, no gait parameters at a usual pace were associated with toe flexor strength. Conversely, walking speed $(\beta=0.22, p=0.049)$,\%swing time $(\beta=0.34, p=0.009)$, and stride length $(\beta=0.22, p=0.011)$ at a fast pace were significantly associated with toe flexor strength.

\section{Discussion}

In the present study, we investigated the association between the toe flexor strength and spatiotemporal gait parameters. After adjustment, the associations were found only in the fast-pace walking condition. Decreased strength of toe flexor was correlated with slower walking speed, shorter periods of single-limb support phase (represented by lower percentage of swing time in gait cycle), and shorter stride length during fast-pace walking. Slower

Table 2 Multivariable regression - cross-sectional effect of toe flexor strength on spatiotemporal gait parameters at usual- and fast-pace walking

\begin{tabular}{llll}
\hline & Adjusted $\boldsymbol{R}^{\mathbf{2}}$ & $\boldsymbol{\beta}$ & $\boldsymbol{p}$ value \\
\hline Usual-pace walking & & & \\
Walking speed (m/s) & 0.07 & 0.10 & 0.456 \\
Cadence (steps/min) & 0.27 & -0.01 & 0.934 \\
\%Swing time & 0.04 & 0.25 & 0.057 \\
Stride length (m) & 0.23 & 0.14 & 0.233 \\
Fast-pace walking & & & \\
Walking speed (m/s) & 0.29 & 0.22 & $0.049^{*}$ \\
Cadence (steps/min) & 0.10 & -0.13 & 0.623 \\
\%Swing time & 0.09 & 0.34 & $0.009^{*}$ \\
Stride length (m) & 0.57 & 0.22 & $0.011^{*}$
\end{tabular}

All models adjusted for age, sex, weight, height, and strength of hand grip. Adjusted $R^{2}=$ proportion of variance in each spatiotemporal gait parameters accounted for by variance in the independent variables; $\beta=$ standardized regression coefficient; $p$ value $=$ Significance of $\beta .{ }^{*} p$ values $<0.05$. walking speed is a sensitive marker of mobility limitation in older adults, and shorter periods of single-limb support phase represent poor balance control during gait $[23,24]$. Thus, these change of spatiotemporal gait parameters indicate deterioration in walking ability. Our results suggest that toe flexor muscles play an important role in walking at a fast pace in older adults.

Fast-pace walking requires generation of high peak power and greater functional reserve compared with usual-pace walking $[13,25]$. It was reported that total generated mechanical work of the lower extremities is more strongly affected by aging at the fast-pace walking condition compared with the usual-pace condition [15]. The toe flexor muscles exert the propulsive force when walking. Thus, walking at a usual pace may not sufficiently require the function of the toe flexor muscles, and the associations between the strength of toe flexor muscles and spatiotemporal gait parameters would be observed in only the fast-pace condition.

The findings of our study are similar to those by Spink et al. in terms of the finding that toe flexor strength was correlated with walking speed [10]. However, the authors reported that hallux plantar flexion strength was a significant and independent predictor of walking speed at normal pace. In the present study, toe flexor strength was associated with walking speed at a fast pace, but not at a usual pace. This discrepancy may depend on the sample characteristics, as Spink's subjects may be frailer as shown by a slower than usual pace walking speed (our study sample: $1.42 \pm 0.19 \mathrm{~m} / \mathrm{s}$ vs. Spink sample: $0.96 \pm 0.20 \mathrm{~m} / \mathrm{s}$ ) and by $11.48 \%$ of subjects using a walking aid outside of the home (in our study no subjects used a walking aid outside of the home). Therefore, walking at usual pace might be challenging for Spink's subjects who had a lower physiological reserve required for function of the toe flexor muscles. Some of the settings in our study were also different from other reports. For example, we investigated the total strength of the toe flexor (hallux and lesser toes) using a foot-gripping force meter with a strain-gauge, while the study by Spink et al. investigated the strength of the hallux flexor and lesser toe flexor separately using hand-held dynamometry. We also performed an adjustment using the anthropometric index (body weight and body height) and other characteristic measures, which may result in our finding of toe flexor strength associated with walking speed at fast pace only.

Our results have potentially important implications for older people. To live an independent life, there are many situations requiring people to walk at faster speeds than usual-pace (e.g., while crossing a street to avoid a collision with an oncoming car or while making a hurried visit to a destination in time). Our results suggest that the maximum speed of community-dwelling older people with poorer toe flexor strength needed in such situations 
was smaller than that of older people with better strength. Furthermore, to our knowledge, this is the first study to indicate the associations between toe flexor strength and spatiotemporal gait parameters including periods of single-limb support phase and stride length, specifically in healthy community-dwelling older adults. We found that a decreased strength of toe flexor was associated with a shorter percentage of single-limb support phase and a shorter stride length during fast-pace walking. Periods of single-limb support phase are related to balance control [24]. Stride length is the major source to change walking speed [24]. These two parameters are fundamental measures that represent human walking features. Thereafter, older people with poor toe flexor strength may experience difficulty walking safely under certain conditions. These results provide further support for an important role of the toe flexor muscle.

There are several explanations for the association between toe flexor strength and spatiotemporal gait parameters. Toe muscle strength has been shown to be associated with balance function (e.g., maximum balance range or coordinated stability) $[9,10,26]$. Subjects with poor toe flexor strength may have more difficulties in maintaining their body stability, especially for single-limb support, which may result in the association between reduced toe flexor strength and shorter periods of single-limb support or slower walking speed. A higher strength of the toe flexors is required for higher push-off forces needed to propel the body farther forward. Menz et al. [12] reported that the plantar flexion strength of the hallux was associated with maximum force and peak pressure under the hallux during the propulsive phase of gait. Thus, a lower strength of toe flexor would be associated with lower push-off forces and lower propulsion of the body further forward, which is related to shorter stride length and slower walking speed.

Our study has some limitations. First, our findings are based on cross-sectional associations, and therefore they do not allow any inference of causal relationships. Future studies are warranted to establish the temporal direction of the relationships between the toe flexor strength and gait parameters. Second, although we were able to control for characteristics likely to modify this relationship, residual potential confounders still may be present, including cognitive function [20] or psychosocial function [27]. However, there are few studies that have investigated the association between gait parameters and strength of toe flexor considering the potential confounding factors. Thus, we believe our results indicating such associations after adjustments for confounding are important. Third, we did not conduct a randomized order of the two walk tests to eliminate a learning effect in completion of the second course (i.e., the fast-pace condition). Many other studies examining gait parameters during various walking speed conditions have used a validated protocol involving usual-pace walking performed first, following by other conditions including fast-pace walking [13,20-22]. Therefore, we used this protocol in the present study.

\section{Conclusion}

Decreased strength of the toe flexor was correlated with slower walking speed, shorter periods of single-limb support phase, and shorter stride length during fast-pace walking. These correlations provide further support for an important role of toe flexor muscles in walking. Additional research is required to establish whether intervention programs that include strengthening exercises for the toe flexor muscle may achieve improvement of spatiotemporal gait parameters.

\section{Abbreviations}

LSA: Life-space assessment; \%swing time: Percent of swing time in gait cycle.

\section{Competing interests}

The authors declare that they have no competing interests.

\section{Authors' contributions}

SM has made substantial contributions to data acquisition, data analysis and interpretation of the data analysis and has been involved in drafting the manuscript. TD has made substantial contributions to interpretation of the data analysis and has been involved in revising the manuscript. TA has made substantial contributions to conception and design of the study, data acquisition, data analysis and interpretation of the data analysis. RS, KT and SN has made substantial contributions to data acquisition, data analysis and interpretation of the data analysis. MY has made substantial contributions to conception and design of the study and data acquisition. RO has made substantial contributions to conception and design of the study and has been involved in revising the manuscript. All authors read and approved the final manuscript.

\section{Acknowledgments}

The authors acknowledge Dr. Soichiro Hirata and Dr. Hiroshi Ando for their valuable advice on methodology and data analysis.

\section{Author details}

${ }^{1}$ Kobe City Hospital Organization, Kobe City Medical Center, West Hospital 2-4 Ichibancho, Nagata-ku, Kobe, Hyogo 653-0013, Japan. ²Department of Community Health Sciences, Kobe University Graduate School of Health Sciences, 7-10-2 Tomogaoka, Suma-ku, Kobe, Hyogo 654-0142, Japan. ${ }^{3}$ Section for Health Promotion, Department for Research and Development to Support Independent Life of Elderly, Center for Gerontology and Social Science, National Center for Geriatrics and Gerontology, 35 Gengo, Morioka, Obu, Aichi 474-8511, Japan. ${ }^{4} J a p a n$ Society for the Promotion of Science, Kojimachi Business Center Building, 5-3-1 Kojimachi, Chiyoda-ku, Tokyo 102-0083, Japan. ${ }^{5}$ Research Institute, National Center for Geriatrics and Gerontology, 35 Gengo, Morioka, Obu, Aichi 474-8511, Japan. ${ }^{6}$ Department of Physical Therapy, Faculty of Rehabilitation, Kobe gakuin University, 518 Arise, Ikawadani-cho, Nishi-ku, Kobe, Hyogo 651-2180, Japan. ${ }^{7}$ Department of Rehabilitation Sciences, Kobe University Graduate School of Health Sciences, 7-10-2 Tomogaoka, Suma-ku, Kobe, Hyogo 654-0142, Japan. ${ }^{8}$ Graduate School of Comprehensive Human Sciences, University of Tsukuba, 3-29-1 Otsuka, Bunkyo-ku, Tokyo 112-0012, Japan.

Received: 12 February 2014 Accepted: 2 October 2014 Published: 8 October 2014

\section{References}

1. Simoneau GG: Kinesiology of Walking. In Kinesiology of the Musculoskeletal System: Foundations for Physical Rehabilitation. 2nd edition. Edited by Neumann DA. St. Louis: Mosby; 2002:627-681. 
2. Mann R, Inman VT: Phasic activity of intrinsic muscles of the foot. J Bone Joint Surg Am 1964, 46:469-481.

3. Jacob HA: Forces acting in the forefoot during normal gait-an estimate. Clin Biomech (Bristol, Avon) 2001, 16:783-792.

4. Menz HB, Zammit GV, Munteanu SE, Scott G: Plantarflexion strength of the toes: age and gender differences and evaluation of a clinical screening test. Foot Ankle Int 2006, 27:1103-1108.

5. Spink MJ, Fotoohabadi MR, Menz HB: Foot and ankle strength assessment using hand-held dynamometry: reliability and age-related differences. Gerontology 2010, 56:525-532.

6. Murata S, Kai Y, Tanaka S, Yamasaki S: Development of a strain gauge based foot-gripping force meter. Rigakuryoho Kagaku 2006, 21:363-367.

7. Mickle KJ, Munro BJ, Lord SR, Menz HB, Steele JR: ISB Clinical Biomechanics Award 2009: toe weakness and deformity increase the risk of falls in older people. Clin Biomech (Bristol, Avon) 2009, 24:787-791.

8. van Schie $\mathrm{CH}$, Vermigli C, Carrington AL, Boulton A: Muscle weakness and foot deformities in diabetes: relationship to neuropathy and foot ulceration in Caucasian diabetic men. Diabetes Care 2004, 27:1668-1673.

9. Menz HB, Morris ME, Lord SR: Foot and ankle characteristics associated with impaired balance and functional ability in older people. J Gerontol A Biol Sci Med Sci 2005, 60:1546-1552

10. Spink MJ, Fotoohabadi MR, Wee E, Hill KD, Lord SR, Menz HB: Foot and ankle strength, range of motion, posture, and deformity are associated with balance and functional ability in older adults. Arch Phys Med Rehabil 2011, 92:68-75.

11. Menz $H B$, Morris $M E$, Lord SR: Foot and ankle risk factors for falls in older people: a prospective study. J Gerontol A Biol Sci Med Sci 2006, 61:866-870

12. Menz HB, Morris ME: Clinical determinants of plantar forces and pressures during walking in older people. Gait Posture 2006, 24:229-236.

13. Annweiler C, Schott AM, Montero-Odasso M, Berrut G, Fantino B, Herrmann FR, Beauchet $O$ : Cross-sectional association between serum vitamin $D$ concentration and walking speed measured at usual and fast pace among older women: the EPIDOS study. J Bone Miner Res 2010, 25:1858-1866.

14. Ko SU, Stenholm S, Chia CW, Simonsick EM, Ferrucci L: Gait pattern alterations in older adults associated with type 2 diabetes in the absence of peripheral neuropathy-results from the Baltimore Longitudinal Study of Aging. Gait Posture 2011, 34:548-552.

15. Ko SU, Hausdorff JM, Ferrucci L: Age-associated differences in the gait pattern changes of older adults during fast-speed and fatigue conditions: results from the Baltimore longitudinal study of ageing. Age Ageing 2010 39:688-694.

16. Kalbe E, Calabrese P, Schwalen S, Kessler J: The Rapid Dementia Screening Test (RDST): a new economical tool for detecting possible patients with dementia. Dement Geriatr Cogn Disord 2003, 16:193-199.

17. Sakai Y, Kotaka A, Murayama N, Takano M, Hirose K, Eto K, Arai H: Japanese version of the: rapid dementia screening test -effectiveness in detecting possible patients with dementia. Jpn J Geriatr Psychiatr 2006, 17:539-551.

18. Baker PS, Bodner EV, Allman RM: Measuring life-space mobility in community-dwelling older adults. J Am Geriatr Soc 2003, 51:1610-1614.

19. Peel C, Sawyer Baker P, Roth DL, Brown CJ, Brodner EV, Allman RM: Assessing mobility in older adults: the UAB study of aging life-space assessment. Phys Ther 2005, 85:1008-1119.

20. Sekiya N, Nagasaki H: Reproducibility of the walking patterns of normal young adults: test-retest reliability of the walk ratio (step-length/step-rate). Gait Posture 1998, 7:225-227.

21. Goutier KM, Jansen SL, Horlings CG, Kung UM, Allum JH: The influence of walking speed and gender on trunk sway for the healthy young and older adults. Age Ageing 2010, 39:647-650.

22. Lowry KA, Lokenvitz N, Smiley-Oyen AL: Age- and speed-related differences in harmonic ratios during walking. Gait Posture 2012, 35:272-276

23. Peel NM, Kuys SS, Klein K: Gait speed as a measure in geriatric assessment in clinical settings: a systematic review. J Gerontol A Biol Sci Med Sci 2013, 68:39-46.

24. Lincoln A: The temporal-spatial parameters. In Clinical Gait Analysis: Theory and Practice. Edited by Kirtley C. New York: Churchill Livingstone; 2006:15-37.

25. Fitzpatrick AL, Buchanan CK, Nahin RL, Dekosky ST, Atkinson HH, Carlson MC, Williamson JD: Associations of gait speed and other measures of physical function with cognition in a healthy cohort of elderly persons. J Gerontol A Biol Sci Med Sci 2007, 62:1244-1251.

26. Endo M, Ashton-Miller JA, Alexander NB: Effects of age and gender on toe flexor muscle strength. J Gerontol A Biol Sci Med Sci 2002, 57:M392-M397.

27. Deshpande N, Metter EJ, Ferrucci L: Sensorimotor and psychosocial correlates of adaptive locomotor performance in older adults. Arch Phys Med Rehabil 2011, 92:1074-1079.

doi:10.1186/1743-0003-11-143

Cite this article as: Misu et al:: Association between toe flexor strength and spatiotemporal gait parameters in community-dwelling older people. Journal of NeuroEngineering and Rehabilitation 2014 11:143.

\section{Submit your next manuscript to BioMed Central and take full advantage of:}

- Convenient online submission

- Thorough peer review

- No space constraints or color figure charges

- Immediate publication on acceptance

- Inclusion in PubMed, CAS, Scopus and Google Scholar

- Research which is freely available for redistribution

Submit your manuscript at www.biomedcentral.com/submit
( Biomed Central 